\title{
Heat evacuation from active Raman media using frequency-selective dissipative coupling
}

\author{
Galina Nemova $\circledast^{1, *}$ and Christophe Caloz $\odot^{1,2}$ \\ ${ }^{1}$ Polytechnique Montréal, 2500 ch. de Polytechnique, Montréal, H3T 1J4, Quebec, Canada \\ ${ }^{2}$ KU Leuven, Kasteelpark Arenberg 10, box 2444, 3001 Leuven, Belgium
}

(Received 10 September 2020; accepted 30 November 2020; published 15 January 2021)

\begin{abstract}
We propose to use frequency-selective dissipative coupling to reduce the heat generated by coherent antiStokes Raman scattering (CARS) reversed cycles in active Raman media with phase mismatch. This is accomplished using a coupled-waveguide structure, which includes the active Raman waveguide (RW), where the Stokes signal undergoes amplification via stimulated Stokes Raman scattering (SSRS), and a dissipative waveguide (DW), which is tuned to the anti-Stokes wavelength so as to evacuate the corresponding anti-Stokes photons from the RW by coupling. The DW introduces optical loss that partially offsets the growth of the anti-Stokes signal in the RW and hence suppress the reversed CARS cycles that would otherwise result into heat generation in the RW. It is shown that the frequency-selective dissipative coupling provided by the DW can reduce the heat in active Raman media by a very factor of up to 5 when the CARS phase mismatch is compensated for by the optimum level of coupling between the RW and the DW.
\end{abstract}

DOI: 10.1103/PhysRevResearch.3.013050

In 1929, Pringsheim proposed to use anti-Stokes fluorescence to cool sodium vapor by leveraging the quantum defect existing between the pump and anti-Stokes photons to annihilate phonons [1]. This approach was later extended to cool solids, such as rare-earth (RE) doped glasses [2] or crystals $[3,4]$ and to mitigate heat generation in RE-doped lasers or Raman lasers [5-9]. In these cases, each cooling cycle includes the absorption of the pump photon followed by phonon annihilation and radiation of an anti-Stokes photon that removes energy from the system. The energy difference between the anti-Stokes and pump photons is equal to the suppressed heat energy. Unfortunately, a reverse cycle that results into heat generation also takes place in the system. The reversed cycle consists of in anti-Stokes photon absorption followed by pump photon emission and phonon generation that reintroduces some energy into the system. These reverse cycles are major obstacles to laser cooling with anti-Stokes fluorescence, and solutions to this issue are needed to advance this technology.

In this work, we propose to use frequency-selective dissipative coupling to suppress the reversed cycles in laser cooled systems, and hence enhance the cooling efficiency. This approach may represent a major advance in the long-lasting issue of laser cooling limitation due to reverse anti-Stokes cycles in different solids. We shall consider its application to Raman active media and specifically show how frequency-

\footnotetext{
*Corresponding author: galina.nemova@yahoo.ca

Published by the American Physical Society under the terms of the Creative Commons Attribution 4.0 International license. Further distribution of this work must maintain attribution to the author(s) and the published article's title, journal citation, and DOI.
}

selective dissipative coupling can improve heat mitigation in Raman amplifiers.

The operation of Raman amplifiers is based on stimulated Stokes Raman scattering (SSRS), which is depicted in Fig. 1(a). SSRS is a third-order nonlinear, inelastic scattering process occurring in certain materials, called Raman media, such as tungstate $\left(\mathrm{BaWO}_{4}[10], \mathrm{SrWO}_{4}[11,12]\right)$, nitrate crystals $\left[\mathrm{Ba}\left(\mathrm{NO}_{3}\right)_{2}\right]$ [13], vanadate $\left(\mathrm{GdVO}_{4}\right)$ [14], silicon [15], diamond [16], etc. As all conventional lasers and amplifiers, Raman amplifiers suffer from heat generation within the active medium caused by the quantum defect between the pump and lasing (Stokes) photons, which deteriorates the device performance. In addition to SSRS, such media involve the processes of coherent anti-Stokes Raman scattering (CARS) and stimulated anti-Stokes Raman scattering (SARS), which are, respectively, represented in Figs. 1(b) and 1(c). SSRS and SARS are processes that generate a single photon and a single phonon per cycle. In contrast, CARS is a four-wave mixing process that exchanges energy with the medium; causing the annihilaton of two phonon per cycle, and may be used for heat reduction [17].

Our enhanced-cooling technique combining anti-Stokes and dissipative coupling is depicted in Fig. 2. Specifically, this technique is based on a frequency-selective dissipative coupling and mitigates heat in Raman active media even in the presence of some phase mismatch. Contrary to conventional planar Raman lasers and amplifiers, which involve a single waveguide, our structure consists of two coupled waveguides. One of these waveguides is the Raman waveguide (RW), which supports nonlinear depleted pump power at the wavelength $\lambda_{p}$ and generation and amplification of the Stokes and anti-Stokes modes at the wavelengths $\lambda_{s}$ and $\lambda_{a}$, respectively, while the second waveguide is a dissipative waveguide (DW) that is tuned to the anti-Stokes wavelength $\lambda_{a}$ to evacuate the related heat energy. The RW-DW coupled pair is designed to 


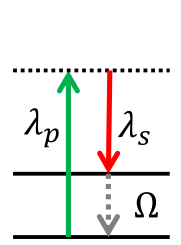

(a)

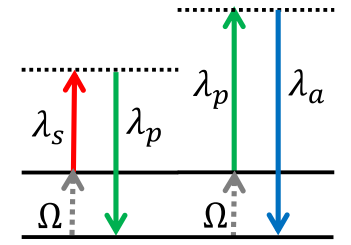

(b)

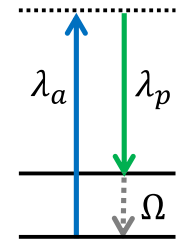

(c)
FIG. 1. Processes taking place in a Raman medium: (a) SSRS, (b) CARS, (c) SARS. $\lambda_{p}, \lambda_{s}, \lambda_{a}$ are the pump, Stokes and anti-Stokes wavelengths, while $\Omega$ is the phonon frequency.

form a system with frequency-selective dissipative coupling where the DW provides an optimal evacuation channel for the anti-Stokes phonon and an efficient strategy to reduce heat. In our scheme frequency-selective dissipative coupling, which is provided by a loss channel made of the DW for the growing anti-Stokes signal in the RW, prevents reverse CARS cycles in the RW accompanied by phonon generation.

The operation of the proposed structure can be analyzed with the propagation equations, which describe the evolution of the electric field of the pump $\left(E_{p}\right)$, Stokes $\left(E_{s}\right)$, anti-Stokes $\left(E_{a}\right)$, and DW $\left(E_{D}\right)$ modes, respectively:

$$
\begin{gathered}
\frac{\partial E_{p}}{\partial z}=-\frac{\lambda_{s}}{\lambda_{p}} G_{s}\left|E_{s}\right|^{2} E_{p}+G_{p}\left|E_{a}\right|^{2} E_{p}-\alpha_{p} E_{p} \\
\frac{\partial E_{s}}{\partial z}=G_{s}\left|E_{p}\right|^{2} E_{s}+\frac{\lambda_{a}}{\lambda_{s}} C_{s a} E_{p}^{2} E_{a}^{*} e^{i \Delta k z}-\alpha_{s} E_{s} \\
\frac{\partial E_{a}}{\partial z}=-G_{p} \frac{\lambda_{p}}{\lambda_{a}}\left|E_{p}\right|^{2} E_{a}-C_{s a} E_{p}^{2} E_{s}^{*} e^{i \Delta k z}+i \kappa_{D} E_{D}-\alpha_{a} E_{a} \\
\frac{\partial E_{D}}{\partial z}=i \kappa_{D} E_{D}-\alpha_{D} E_{D} \\
G_{s}=\frac{1}{4} \sqrt{\frac{\varepsilon_{0}}{\mu_{0}}} g_{R}, \quad G_{p}=\frac{\lambda_{s}}{\lambda_{p}} G_{s}, \quad C_{s a}=\frac{\lambda_{s}}{\lambda_{a}} G_{s}
\end{gathered}
$$

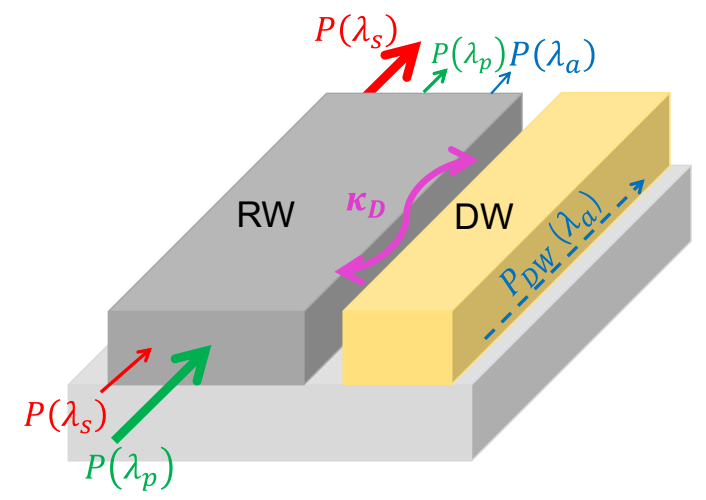

FIG. 2. Proposed frequency-selective dissipative structure to enhance the cooling efficiency of a Raman amplifier, composed of a Raman waveguide (RW), where the generation of the Stokes and anti-Stokes signals takes place, and a dissipative waveguide (DW), that is tuned to the anti-Stokes wavelength to evacuate and dissipate the anti-Stoke photons. $\kappa_{D}$ is the coupling coefficient between the waveguides. where $E_{p}, E_{s}, E_{a}$, and $E_{D}$ are the complex amplitudes of the pump, Stokes, anti-Stokes, and DW modes, respectively, $\kappa_{D}$ is the coupling coefficient between the RW and the DW, $\alpha_{p}, \alpha_{s}$, and $\alpha_{a}$ are the absorption coefficients in the RW at the pump, Stokes, and anti-Stokes wavelengths, respectively. $\alpha_{D}$ is the optical loss in the DW at the anti-Stokes wavelength $\lambda_{a} . g_{R}$ is the Raman gain coefficient. $\Delta k=|\Delta \vec{k}|=$ $\left|2 \overrightarrow{k_{p}}-\overrightarrow{k_{s}}-\overrightarrow{k_{a}}\right|$ is the mismatch, which includes the wave vectors of the pump $\left(\overrightarrow{k_{p}}\right)$, Stokes $\left(\overrightarrow{k_{s}}\right)$, and anti-Stokes $\left(\overrightarrow{k_{a}}\right)$ modes. If $\Delta k=0$ the CARS process is phase matched. If $\Delta k \neq 0$ it is mismatched. If we put the relations $E_{s}(z)=$ $B_{s}(z) e^{i \Delta k z / 2}, E_{a}(z)=B_{a}(z) e^{i \Delta k z / 2}$, and $E_{D}(z)=B_{D}(z) e^{i \Delta k z / 2}$ into equations of the system (1)-(4), we will see that the CARS process is quasiphase matched if $|\Delta k| \ll G_{s}\left|E_{p}\right|^{2}$. Taking into account the relations $P_{p, s, a}=\frac{1}{2}\left(\frac{\varepsilon_{0}}{\mu_{0}}\right)^{\frac{1}{2}}\left|E_{p, s, a}\right|^{2} A_{\mathrm{eff}}^{R W}$, $P_{D}=\frac{1}{2}\left(\frac{\varepsilon_{0}}{\mu_{0}}\right)^{\frac{1}{2}}\left|E_{D}\right|^{2} A_{\text {eff }}^{D W}$, and $N_{p, s, a, D}=\frac{P_{p, s, a, D}}{h v_{p, s, a, D}}$ and using the system of Eqs. (1)-(4) one obtains the system of equations, which describe the evolution of the number of photons in the pump $\left(N_{p}\right)$, Stokes $\left(N_{s}\right)$, and anti-Stokes $\left(N_{a}\right)$ modes of the RW as well as the evolution of the number of photons at the anti-Stokes wavelength $\lambda_{a}$ in the mode propagating in the DW $\left(N_{D}\right)$ along the axis $(z)$ of the coupled structure:

$$
\begin{gathered}
\frac{\partial N_{p}}{\partial z}=\frac{A_{\mathrm{eff}}^{R W}}{\mu_{0} h c^{2}} \lambda_{p}\left[-\frac{\lambda_{s}}{\lambda_{p}} G_{s}\left|E_{s}\right|^{2}\left|E_{p}\right|^{2}\right. \\
\left.+G_{p}\left|E_{a}\right|^{2}\left|E_{p}\right|^{2}-\alpha_{p}\left|E_{p}\right|^{2}\right], \\
\frac{\partial N_{s}}{\partial z}=\frac{A_{\mathrm{eff}}^{R W}}{\mu_{0} h c^{2}} \lambda_{s}\left[G_{s}\left|E_{s}\right|^{2}\left|E_{p}\right|^{2}+\frac{\lambda_{a}}{\lambda_{s}} \widetilde{C_{s a}}-\alpha_{s}\left|E_{s}\right|^{2}\right], \\
\frac{\partial N_{a}}{\partial z}=\frac{A_{\mathrm{eff}}^{R W}}{\mu_{0} h c^{2}} \lambda_{a}\left[-G_{p} \frac{\lambda_{p}}{\lambda_{a}}\left|A_{a}\right|^{2}\left|E_{p}\right|^{2}\right. \\
\left.-\widetilde{C_{s a}}+\widetilde{C_{R D}}-\alpha_{a}\left|A_{a}\right|^{2}\right], \\
\frac{\partial N_{D}}{\partial z}=\frac{A_{\mathrm{eff}}^{D W}}{\mu_{0} h c^{2}} \lambda_{a}\left[-\widetilde{C_{R D}}-\alpha_{D}\left|A_{D}\right|^{2}\right],
\end{gathered}
$$

where $A_{a, D}=E_{a, D} e^{-i \kappa_{D}}, A_{\text {eff }}^{R W}$ and $A_{\text {eff }}^{D W}$ are the effective areas of the RW and DW, respectively,

$$
\widetilde{C_{s a}}=\operatorname{Re}\left(C_{s a} E_{p}^{2} A_{a}^{*} E_{s}^{*} e^{i\left(\Delta k-\kappa_{D}\right) z}\right), \quad \widetilde{C_{R D}}=\operatorname{Re}\left(i \kappa_{D} A_{D} A_{a}^{*}\right) .
$$

Let us consider the different terms in Eqs. (6)-(9) in some detail. The terms with $G_{s}$ and $G_{p}$ are related to the process of stimulated Raman scattering. The first terms in the square brackets of Eqs. (7) and (8) describe the change in the number of the Stokes and anti-Stokes photons propagating in the RW as a result of the SSRS and SARS processes, respectively. Both these processes are accompanied by phonon generation [Figs. 1(a) and 1(c)]. Each of the SSRS or SARS cycles generates a single phonon with the energy $h \Omega$ resulting into heating of the RW. The terms $\widetilde{C_{R D}}$ in Eqs. (8) and (9) describe the change in the anti-Stokes photon numbers in the RW and DW caused by the mutual coupling of these waveguides. The terms $\widetilde{C_{s a}}$ in (7) and (8) are related to the CARS process [Fig. 1(b)]. They include the phase mismatch of the CARS 
process $\Delta \vec{k} \cdot \tilde{\mathrm{z}}=\left(2 \overrightarrow{k_{p}}-\overrightarrow{k_{s}}-\overrightarrow{k_{a}}\right) \cdot \vec{z}$, which depends on the wave vectors of the pump $\left(\overrightarrow{k_{p}}\right)$, Stokes $\left(\overrightarrow{k_{s}}\right)$, and anti-Stokes $\left(\overrightarrow{k_{a}}\right)$ modes. The terms $\widetilde{C_{s a}}$ include the phase change $\kappa_{D} z$ that is caused by the coupling between the RW and the DW. This phase change can compensate for the phase mismatch $\Delta \vec{k} \cdot \tilde{z}$. As one can infer from Fig. 1(b), the CARS process can take place only in an excited Raman medium because it is triggered by photons that are generated by SSRS. As can be seen in Eq. (8), the CARS process alternatively serves as a source for anti-Stokes photon generation in the RW.

If the RW and the DW are not coupled $\left(\kappa_{D}=0\right)$ and phase matching is insufficient to satisfy the relation $|\Delta k| \ll G_{s}\left|E_{p}\right|^{2}$, the coefficient $\widetilde{C_{s a}}$ oscillates and changes its sign along the length of the sample. As we already mentioned Eq. (8) describes the increase $\left(\widetilde{C_{s a}}<0\right)$ or decrease $\left(\widetilde{C_{s a}}>0\right)$ in the anti-Stokes phonon number along the length of the sample as a result of CARS. The decrease in the anti-Stokes phonon number is caused by reversed CARS cycles when all the arrows in Fig. 1(b) reverse their direction. This reverse CARS process, which starts with the anti-Stokes photon absorption, generates thus a Stokes photon and two phonons. These phonons are sources of heat and therefore alter the cooling efficiency of the system. The role of the DW is to remove these CARSgenerated anti-Stokes photons by the introduction of loss. But the problem is not trivial. If the DW is lossless $\left(\alpha_{D}=0\right)$, the anti-Stokes signal oscillates between the RW and the DW along the coupled structure with a period corresponding to the coherence length. The loss $\left(\alpha_{D} \neq 0\right)$ in the DW prevents such oscillation, but it must still be small enough to provide a sufficient coupling length for the initial transfer of the anti-Stokes phonons into it. The loss of the DW should therefore be tuned to the optimum accounting for these two antagonistic effects, the ultimate goal being to maximize the amount of phonon power dissipation in the RW. This power can be estimated as the difference between the phonon power generated by SSRS plus SARS and the phonon power annihilated or generated by CARS or reverse CARS, respectively. Each SSRS and SARS cycle generate a single phonon [Figs. 1(a) and 1(c)]. The first terms in the square brackets in Eq. (7) and (8) describe the number of phonons generated with SSRS and SARS, respectively. The number of phonons generated in the RW at the $z$ point with both cycles is

$$
\begin{aligned}
N_{\text {Phonon }}^{\left(S S R S_{-} S A R S\right)} & =\frac{A_{\text {eff }}^{R W}}{\mu_{0} h c^{2}} \lambda_{s} G_{s}\left|E_{s}\right|^{2}\left|E_{p}\right|^{2}+\frac{A_{\text {eff }}^{R W}}{\mu_{0} h c^{2}} \lambda_{p} G_{p}\left|E_{p}\right|^{2}\left|A_{a}\right|^{2} \\
& =\frac{A_{\text {eff }}^{R W}}{\mu_{0} h c^{2}} \lambda_{s} G_{s}\left|E_{p}\right|^{2}\left(\left|E_{s}\right|^{2}+\left|A_{a}\right|^{2}\right) .
\end{aligned}
$$

The term $\widetilde{C_{s a}}$ in Eqs. (7) and (8) describe the number of phonons annihilated or generated in the RW with CARS or reverse CARS cycles, respectively. The number of phonons associated with the CARS cycles on at the $z$ point is

$$
\begin{aligned}
N_{\text {Phonon }}^{\left(S S R_{S} S A R S_{-}-C A R S\right)} & =2 \frac{A_{\mathrm{eff}}^{R W}}{\mu_{0} h c^{2}} \lambda_{a} C_{s a} \operatorname{Re}\left(E_{p}^{2} A_{a}^{*} E_{s}^{*} e^{i\left(\Delta k-\kappa_{D}\right) z}\right) \\
& =2 \frac{A_{\mathrm{eff}}^{R W}}{\mu_{0} h c^{2}} \lambda_{s} G_{s} \operatorname{Re}\left(E_{p}^{2} A_{a}^{*} E_{s}^{*} e^{i\left(\Delta k-\kappa_{D}\right) z}\right) .
\end{aligned}
$$

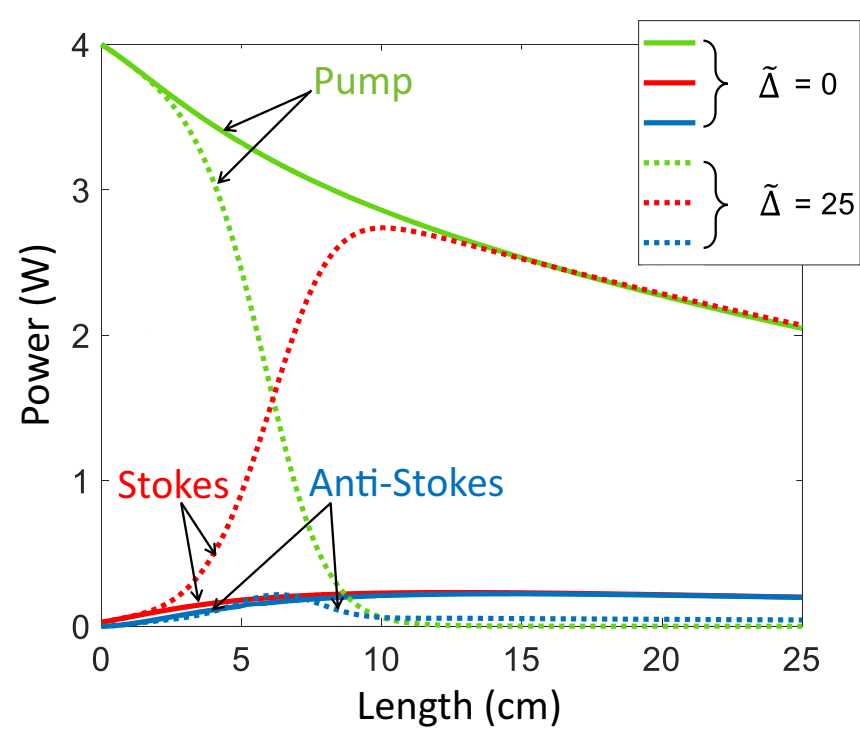

FIG. 3. Power distribution for the pump, Stokes and anti-Stokes signals along the length of the RW without coupling $\left(\kappa_{D}=0\right)$, for the phase matched $(\widetilde{\Delta}=0)$ and phase mismatched $\widetilde{\Delta}=25$ cases.

Here 2 means that each CARS or reverse CARS cycle is accompanied by annihilation or generation of two phonons, respectively. Using relations (12) and (13) we can estimate the phonon power in the system as

$$
\begin{aligned}
P_{\text {phon }}= & h \Omega \frac{A_{\text {eff }}^{R W}}{\mu_{0} h c^{2}} \lambda_{s} G_{s}\left[\left|E_{p}\right|^{2}\left(\left|E_{s}\right|^{2}+\left|A_{a}\right|^{2}\right)\right. \\
& \left.-2 \operatorname{Re}\left(E_{p}^{2} A_{a}^{*} E_{s}^{*} e^{i\left(\Delta k-\kappa_{D}\right) z}\right)\right],
\end{aligned}
$$

where $h \Omega=h c\left(\lambda_{a}^{-1}-\lambda_{p}^{-1}\right)$ is the phonon energy. As an example, let us consider a diamond sample operating as a Raman amplifier. The RW is pumped at the wavelength $\lambda_{p}=1064 \mathrm{~nm}$. The Raman gain coefficient in diamond at $1064 \mathrm{~nm}$ is $g_{R} \approx 10 \mathrm{~cm} / \mathrm{GW}$. The wavelengths of the Stokes and anti-Stokes signals are $\lambda_{s}=1240 \mathrm{~nm}$ and $\lambda_{a}=888 \mathrm{~nm}$, respectively. The absorption loss in the sample at the Stokes and anti-Stokes wavelengths are $\alpha_{s}=\alpha_{a} \approx 0.11 \mathrm{~cm}^{-1}$ [18]. The length of the sample considered in our simulations is $L=25 \mathrm{~cm}$. As we already mentioned, the power of the pump $\left(P_{p}\right)$, Stokes $\left(P_{s}\right)$, and anti-Stokes $\left(P_{a}\right)$ modes propagating in the RW and the power of the waveguide mode propagating in the DW at the anti-Stokes wavelength $\left(P_{D}\right)$ are related to the photon numbers, $N_{p, s, a, D}$, in these modes as $P_{p, s, a, D}=$ $h c N_{p, s, a, D} / \lambda_{p, s, a, D}$, where $h$ is the Planck constant and $c$ is the speed of light in vacuum. The forthcoming numerical results simulate the different power distributions using Eqs. (6)-(9) while Eq. (13) can be used to simulate the phonon distribution along the structure.

Let us first consider the case where the RW and the DW would not be coupled $\left(\kappa_{D}=0\right)$ and the CARS process in the RW would be phase matched $(\Delta k=0)$. The solid curves in Fig. 3 show the power distributions of the pump, Stokes and anti-Stokes signals in solid lines along the RW. The pump power decreases along the sample, due to power transfer to the Stokes and anti-Stokes signals, and the powers of the Stokes and anti-Stokes signals are almost equal to each other. The 


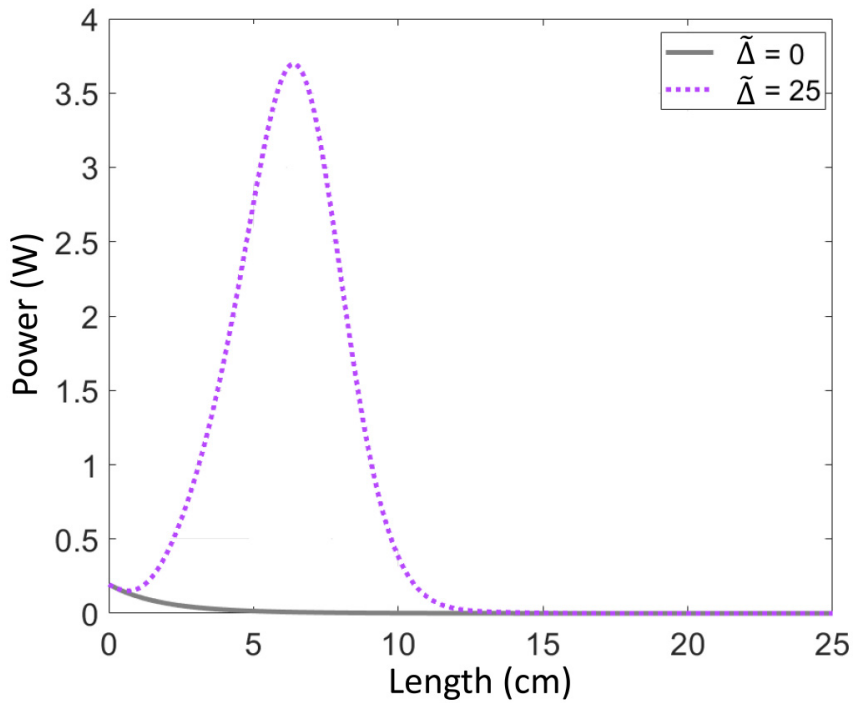

FIG. 4. Phonon power distribution along the RW without coupling $\left(\kappa_{D}=0\right)$ and with phase matching $(\tilde{\Delta}=0)$ and phase mismatch $\widetilde{\Delta}=25$.

solid curve in Fig. 4 shows the corresponding phonon power variation. We see that in the phase matched case, where the reverse CARS cycles are absent, the CARS process annihilates most of the phonons generated by SSRS and SARS.

However, such a perfect matching condition $(\Delta k=0)$ is typically unrealistic in practice. Let us therefore introduce some phase mismatch into the system. For simplicity, we define the normalized phase parameter $\tilde{\Delta}=\Delta k L$. For example, let us consider a system with the normalized phase mismatch $\tilde{\Delta}=25$. In this case, the length of the sample $(L=25 \mathrm{~cm})$ includes several coherent lengths $\left(l_{c} \cong 3 \mathrm{~cm}\right)$. The evolution of the powers in the pump, Stokes, and anti-Stokes signals along this mismatched RW are plotted in dotted lines in Fig. 3. Now, the powers of the Stokes and anti-Stokes signals are

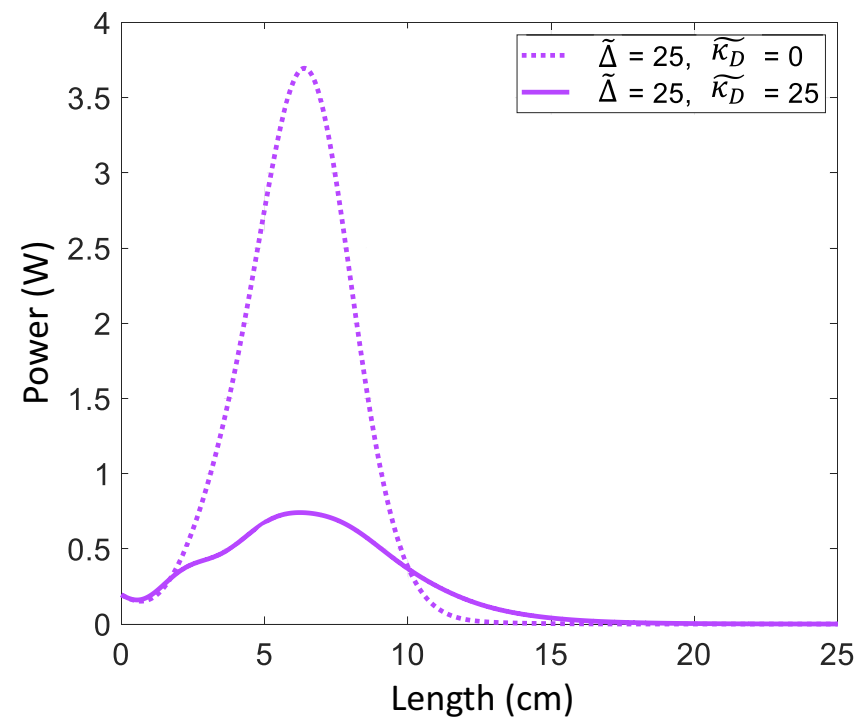

FIG. 5. Phonon power distribution along the RW without coupling $\left(\widetilde{\kappa_{D}}=0\right)$ and with coupling $\widetilde{\kappa_{D}}=25$ and with phase mismatch $\widetilde{\Delta}=25$.

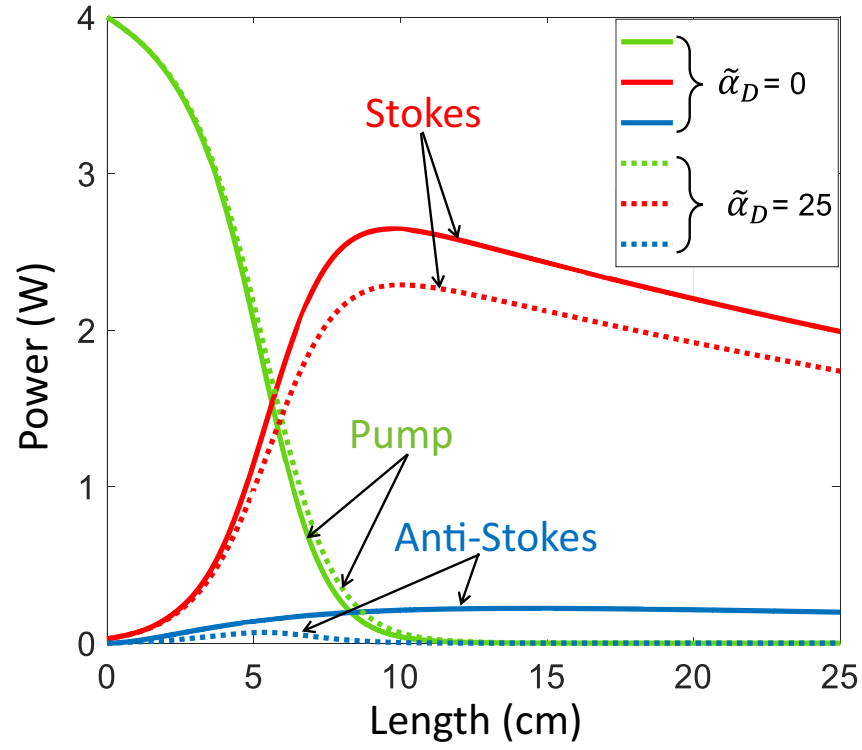

FIG. 6. Power distributions of the pump, Stokes, and anti-Stokes signals along the RW with coupling $\left(\widetilde{\kappa_{D}}=40\right)$ and phase mismatch $(\widetilde{\Delta}=25)$, without loss $\left(\widetilde{\alpha_{D}}=0\right)$ and with loss $\widetilde{\alpha_{D}}=25$.

very different from each other for most structure lengths. The reverse CARS cycles together with the dramatic growth of the Stokes signal result in the dramatic growth of the phonon power in the RW, as shown by the dotted curve in Fig. 4.

As one can see in Eq. (8), the phase mismatch in the system can be compensated for by introducing coupling, $\kappa_{D} \neq 0$. For simplicity, and consistency with the normalized phase, we define the normalized coupling constant $\tilde{\kappa_{D}}=\kappa_{D} L$. Let us consider a system with $\widetilde{\kappa_{D}}=25$, so as to fully compensate for the phase mismatch of $\widetilde{\Delta}=25$. As one can see in Fig. 5, major heat suppression is achieved in this case, the phonon power

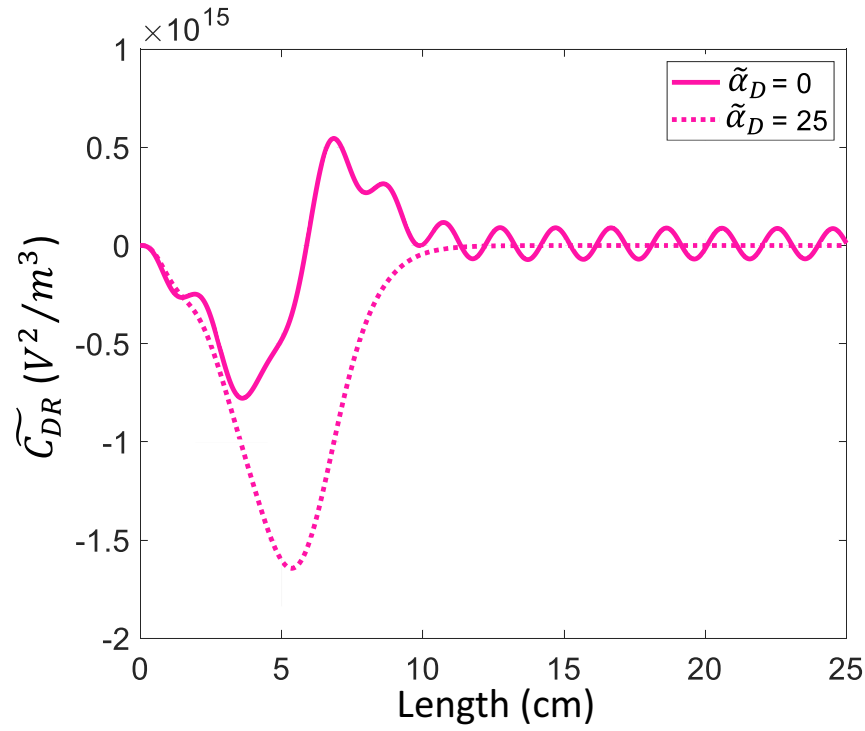

FIG. 7. $\widetilde{C_{D R}}$ parameter along the structure with coupling $\widetilde{\kappa_{D}}=$ 40 and phase mismatched $\widetilde{\Delta}=25$, without loss $\left(\widetilde{\alpha_{D}}=0\right)$ and with loss $\widetilde{\alpha_{D}}=25$. Negative and positive values represent, respectively, phonon annihilation and generation. 


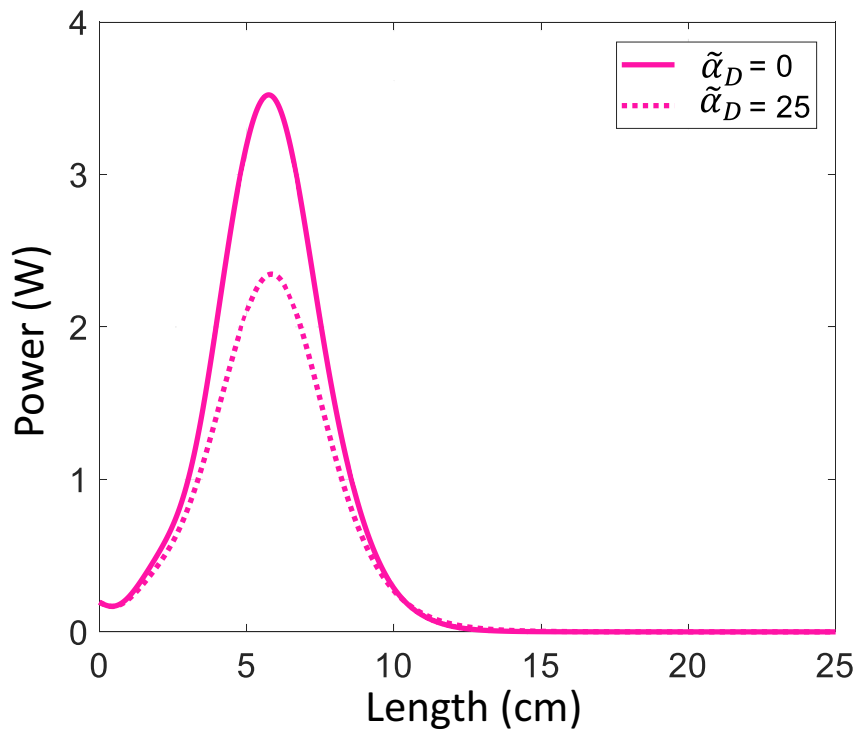

FIG. 8. Phonon power distribution along the RW with coupling $\widetilde{\kappa_{D}}=40$ and phase mismatch $\widetilde{\Delta}=25$, without loss $\left(\widetilde{\alpha_{D}}=0\right)$ and with loss $\widetilde{\alpha_{D}}=25$.

being decreased from $\sim 3.7 \mathrm{~W}$ (dashed curve) to $\sim 0.55 \mathrm{~W}$ (solid curve). Unfortunately, realizing coupled waveguides to provide such perfect compensation is not a simple task. Let us therefore consider the more realistic system where the phase mismatch can be only partially compensated for by coupling. As an example, we set phase mismatch $\tilde{\Delta}=25$ and coupling $\widetilde{\kappa_{D}}=40$, which does not compensate for the phase mismatch, and we compare two designs, one with DW free from any optical loss, i.e., $\widetilde{\alpha_{D}}=\alpha_{D} L=0$, and one with an of optical loss $\widetilde{\alpha_{D}}=25$. The power distributions along this structure are plotted in Fig. 6. In the absence of DW loss $\left.\widetilde{\left(\alpha_{D}\right.}=0\right)$, the power of the anti-Stokes signal is higher than that with loss. Indeed, in the former case, the waveguide coupling constant $\widetilde{C_{R D}}$ oscillates between positive and negative values along the structure, as shown by the solid curve in Fig. 7. Unfortunately, these recoupled anti-Stokes photons can cause reverse CARS and SARS, resulting into the heat generation in the RW. The phonon power generated in the RW when the optical loss in the DW are absent is shown again, for comparison, the solid line in Fig. 8, with a peak of $\sim 3.7 \mathrm{~W}$ [a factor 2.4) or a decrease of structure (Fig. 6), the waveguide coupling constant $\widetilde{C_{R D}}$ becomes exclusively negative (Fig. 7). The majority of the anti-Stokes photons propagating in the DW are thus dissipated in the DW before having a chance of returning to the RW and generating heat there via reverse CARS and SARS. As a result, the phonon power in the system has decreased

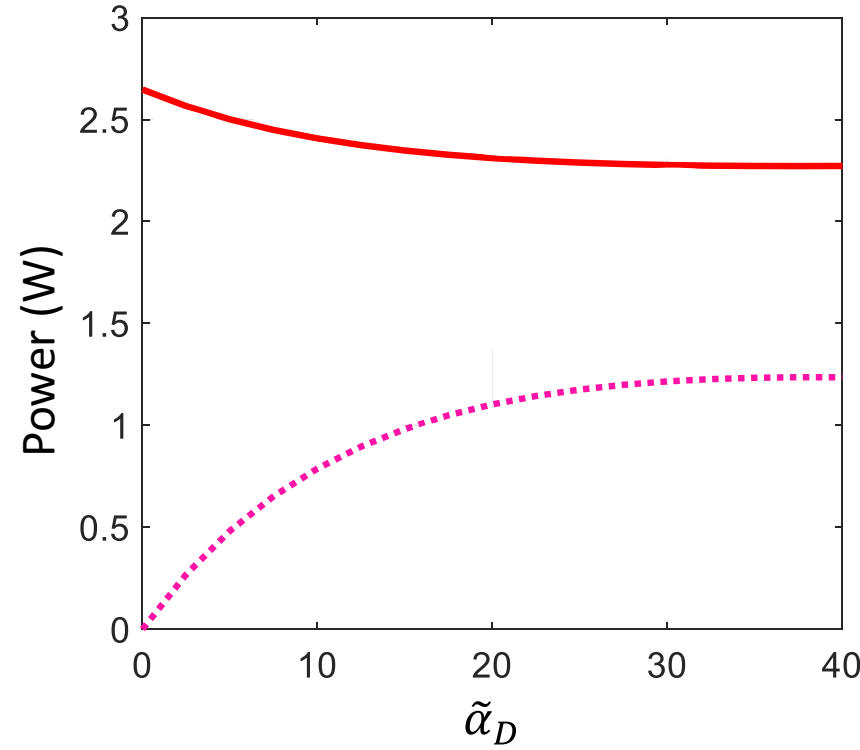

FIG. 9. Evolution of the system performance versus the DW loss $\left(\widetilde{\alpha_{D}}\right)$ for coupling $\widetilde{\kappa_{D}}=40$ and phase mismatch $\widetilde{\Delta}=25$. The dotted curve represents the difference in the phonon peak power in the RW without loss and with loss (Fig. 8 at $\widetilde{\Delta}=25$ ), while the solid curve represents the Stokes power in the RW.

from $\sim 3.7 \mathrm{~W}$ to $\sim 2.4 \mathrm{~W}$ (a factor of 1.4 or a decrease of $35 \%$ ), as shown by the dotted curve in Fig. 8 .

Finally, Fig. 9 represents the evolution of the system performance in terms of the DW loss. While the phonon power reduction monotonically increases with increasing DW loss, this loss unavoidably also affects the power level of the Stokes photons, and hence the power level of the overall device. If stability is the primary concern in the application considered, one may introduce a large amount of loss in the DW (e.g., here $\widetilde{\alpha_{D}}=30$ ); if, in contrast, in the output power level is also an important parameter, one may trade off some stability for higher power by operating in a lower loss regime (e.g., here $\widetilde{\alpha_{D}}=5$ ).

In summary, we have introduced the concept of frequencyselective dissipative coupling in order to improve cooling of active Raman waveguide systems. This concept consists of coupling a dissipative waveguide to the amplification waveguide for optimal phonon suppression. We have illustrated this concept with the example of Stokes amplification in Raman amplifiers, where heat reduction by a factor of more than 2 has been demonstrated in the case of a phase matched system amplifier. This concept represents an alternative approach of anti-Stokes cooling and may play a significant role in the development of more stable and athermal amplifiers and lasers.
[1] P. Pringsheim, Zwei bemerkungen riber den unterschied von lumineszenz und temperature-strahlung, Z. Phys. 57, 739 (1929).

[2] J. V. Guiheen, C. D. Haines, G. H. Sigel, R. I. Epstein, J. Thiede, and W. M. Patterson, $\mathrm{Yb}^{3+}$ and $\mathrm{Tm}^{3+}$ doped fluoroaluminate glasses for anti-Stokes cooling, Phys. Chem. Glasses Eur. J. Glass Sci. Technol. B 47, 167 (2006).
[3] S. Rostami, A. R Albrecht, A. Volpi, and M. Sheik-Bahae, Observation of optical refrigeration in a holmium-doped crystal, Photonics Res. 7, 445 (2019).

[4] E. Soares de Lima Filho, K. V. Krishnaiah, Y. Ledemi, Ye-Jin Yu, Y. Messaddeq, G. Nemova, and R. Kashyap, Ytterbiumdoped glass-ceramics for optical refrigeration, Opt. Express 23, 4630 (2015). 
[5] G. Nemova and R. Kashyap, Ytterbium-doped glass-ceramics for optical refrigeration, J. Lightwave Technol. 27, 5597 (2009).

[6] G. Nemova and R. Kashyap, Fiber amplifier with integrated optical cooler, J. Opt. Soc. Am. B 26, 2237 (2009).

[7] E. Mobini, M. Peysokhan, B. Abaie, and A. Mafi, Thermal modeling, heat mitigation, and radiative cooling for double-clad fiber amplifiers, J. Opt. Soc. Am. B 35, 2484 (2018).

[8] M. Peysokhan, E. Mobini, A. Allahverdi, B. Abaie, and A. Mafi, Characterization of Yb-doped ZBLAN fiber as a platform for radiation-balanced lasers, Photonics Res. 8, 202 (2020).

[9] N. Vermeulen, C. Debaes, P. Muys, and H. Thienpont, Mitigating Heat Dissipation in Raman Lasers Using Coherent Anti-Stokes Raman Scattering, Phys. Rev. Lett. 99, 093903 (2007).

[10] X. H. Chen, P. Li, X. Y. Zhang, Q. P. Wang, Z. J. Liu, Z. H. Cong, L. Li, and H. J. Zhang, Eye-safe Raman laser at $1532 \mathrm{~nm}$ with $\mathrm{BaWO}_{4}$ crystal, Laser Phys. 21, 2040 (2011).

[11] H. Zhang, P. Li, Q. Wang, Q. Wang, X. Chen, X. Zhang, J. Chang, and X. Tao, High-power dual-wavelength eye-safe ceramic Nd:YAG/SrWO ${ }_{4}$ Raman laser operating at 1501 and 1526 nm, Appl. Optics 53, 7189 (2014).
[12] H. Zhang $\mathrm{H}$ and $\mathrm{P}$. Li, High-efficiency eye-safe $\mathrm{Nd}: \mathrm{YAG} / \mathrm{SrWO}_{4}$ Raman laser operating at $1664 \mathrm{~nm}$, Appl. Phys. B 122, 12 (2016).

[13] P. V. Shpak, S. V. Voitikov, R. V. Chulkov, P. A. Apanasevich, V. A. Orlovich, A. S. Grabtchikov, A. Kushwaha, N. Satti, L. Agrawal, A. K. Maini, and A. K. Maini, Passively Q-switched diode-pumped Raman laser with third-order Stokes eye-safe oscillation, Opt. Commun. 285, 3659 (2012).

[14] M. Frank, S. N. Smetanin, M. Jelínek Jr., D. Vyhlídal, V. E. Shukshin, P. G. Zverev, and V. Kubeček, 860 fs $\mathrm{GdV}_{\mathrm{O} 4}$ Raman laser at $1228 \mathrm{~nm}$ pumped by $36 \mathrm{ps}, 1063 \mathrm{~nm}$ laser, Laser Phys. Lett. 16, 085401 (2019).

[15] T. Yasuda, M. Okano, M. Ohtsuka, M. Seki, N. Yokoyama, and Y. Takahashi, Raman silicon laser based on a nanocavity fabricated by photolithography, OSA Continuum 3, 814 (2020).

[16] K. Chrysalidis, V. N. Fedosseev, B. A. Marsh, R. P. Mildren, D. J. Spence, K. D. A. Wendt, S. G. Wilkins, and E. Granados, Granados, Continuously tunable diamond Raman laser for resonance laser ionization, Opt. Lett. 44, 3924 (2019).

[17] B. Bobbs and C. Warner, Raman-resonant four-wave mixing and energy transfer, J. Opt. Soc. Am. B 7, 234 (1990).

[18] Y. Li, Z. Bai, H. Chen, D. Jin, X. Yang, Y. Qi, J. Ding, Y. Wang, and Z. Lu, Eye-safe diamond Raman laser, Results Phys. 16 102853 (2020). 